\title{
Efecto hepatoprotector del extracto hidroetanólico atomizado del maíz morado (Zea mays $L$.) en lesiones hepáticas inducidas en ratas
}

Hepatoprotective effect of atomized hydroethanolic extract from Andean purple corn (Zea mays L.) in induced hepatic injury in rats

\author{
Renán Hañari-Quispe ${ }^{1}$, Jorge Arroyo ${ }^{2,3}$, Oscar Herrera-Calderón ${ }^{1,4}$, \\ Hernán Herrera-Moran \\ Unidad de Posgrado, Facultad de Farmacia y Bioquímica, Universidad Nacional Mayor de San Marcos, Lima, Perú. \\ ${ }^{2}$ Laboratorio de Farmacología Experimental, Facultad de Medicina Humana, Universidad Nacional Mayor de San Marcos, Lima, Perú. \\ ${ }^{3}$ Instituto de Investigaciones Clínicas, Universidad Nacional Mayor de San Marcos, Lima, Perú. \\ ${ }^{4}$ Laboratorio de Fisiología y Fisiopatología, Facultad de Farmacia y Bioquímica, Universidad Nacional San Luis Gonzaga, Ica, Perú.
}

\begin{abstract}
Resumen
Objetivos: Evaluar el efecto hepatoprotector del extracto hidroetanólico atomizado de Zea mays variedad morado sobre lesiones hepáticas inducidas en ratas. Diseño: Experimental. Institución: Laboratorio de Farmacología Experimental, Facultad de Medicina Humana, Universidad Nacional Mayor de San Marcos, Lima, Perú. Material biológico: Extracto hidroetanólico atomizado de maíz morado (EAM). Intervenciones: Se formó seis grupos de ratas machos ( $n=10$, por cada grupo), se les administró fenobarbital a concentración de $0,5 \mathrm{~g} / \mathrm{L}$ en agua potable ad líbitum por 15 días; posteriormente se administró tetracloruro de carbono $(\mathrm{CCl})$ a una dosis de $0,2 \mathrm{~mL} / \mathrm{kg}$, por via oral. El diseño experimental fue el siguiente: G1: suero fisiológico (SSF); G2: CCl $0,2 \mathrm{~mL} / \mathrm{kg}(\mathrm{T}){ }^{4} \mathrm{G} 3: \mathrm{T}+$ silimarina $25 \mathrm{mg} / \mathrm{kg} ; \mathrm{G} 4: T$ + EAM $500 \mathrm{mg} / \mathrm{kg} ; \mathrm{G} 5: T$ + EAM $1000 \mathrm{mg} / \mathrm{kg} ; \mathrm{G} 6: T$ + EAM $2000 \mathrm{mg} / \mathrm{kg}$. Principales medidas de resultados: Perfil hepático, especies reactivas al ácido tiobarbitúrico (TBARS) en suero, índice hepático, observación histológica. Resultados: Se observó aumento significativo $(p<0,05)$ en la actividad de alanina amino transferasa (ALT) entre el grupo $G 2$ y los grupos $G 3$ y $G 4$ $(p<0,001)$. Hubo disminución significativa $(p<0,05)$ de fosfatasa alcalina ( $F A L)$ en el grupo $G 2$ con respecto $G 1$. El nivel de TBARS fue menor en el grupo que recibió $1000 \mathrm{mg} / \mathrm{kg}$ de EAM con respecto al control. Las actividades de HDL-C y triglicéridos no mostraron diferencias significativas. Se ha observado la reducción de $60 \%$ de la lesión hepática, evidenciado con menor daño del hepatocito al estudio histológico. Conclusiones: El extracto hidroetanólico atomizado de maíz morado a la dosis de $1000 \mathrm{mg} / \mathrm{kg}$ disminuyó las lesiones hepáticas inducidas en ratas.
\end{abstract}

Palabras clave: Zea mays L, lesión hepática, hepatomegalia, planta medicinal.

\section{Abstract}

Objectives: To evaluate the hepatoprotector effect of hydroalcoholic extract atomized of Zea mays against hepatic injuries induced in rats. Design: Experimental. Institution: Laboratory of Experimental Pharmacology, Faculty of Human Medicine, Universidad Nacional Mayor de San Marcos, Lima, Peru. Biological material: Hydroethanolic extract atomized of purple corn (EAM). Interventions: To six groups of male rats $(n=10$, for each group) phenobarbital $0.5 \mathrm{~g} / \mathrm{L}$ in drinkable water ad libitum was administered for 15 days, followed by carbon tetrachloride $0.2 \mathrm{~mL} / \mathrm{kg}$ p.o. The experimental design was a follow: G1: normal; G2: CCl $0.2 \mathrm{~mL} / \mathrm{kg}$ (T); G3:T+ silimarina 25 $\mathrm{mg} / \mathrm{kg} ; \mathrm{G} 4:$ T+ EAM $500 \mathrm{mg} / \mathrm{kg} ; \mathrm{G} 5:$ T + EAM $1000 \mathrm{mg} / \mathrm{kg} ; \mathrm{G} 6 \mathrm{~T}+\mathrm{EAM} 2000 \mathrm{mg} / \mathrm{kg}$. Main outcome measures: Hepatic profile, reactive species to thiobarbituric acid (TBARS), liver index, and histological observation. Results: Significant increase $(p<0.05)$ in ALT activity was observed between group $G 2$ in comparison to groups $G 3$ and $G 4(p<0,001)$. There was significant decrease $(p<0.05)$ of alkaline phosphatase (AP) in group G2 with respect to G1. TBARS level was less in the group that received $1000 \mathrm{mg} / \mathrm{kg}$ of EAM regarding to control. HDL-C and triglycerides activities did not show significant differences. Reduction in $60 \%$ of hepatic injury was observed, evidencing less damage of the hepatocyte by histological study. Conclusions: The purple corn atomized hydroethanolic extract at 1000 $\mathrm{mg} / \mathrm{kg}$ dose decreased induced hepatic injuries in rats.

Keywords: Zea mays L, liver injury, hepatomegaly, medicinal plant.

An Fac med. 2015;76(2):123-8 / dx.doi.org/10.15381/anales.v76i2.11136

\section{INTRODUCCIÓN}

La hepatotoxicidad está relacionada a la disfunción o daño asociado a una sobredosis de drogas o xenobióticos. El hígado es altamente susceptible a efectos adversos de los xenobióticos propios de su rol principal en el metabolismo y excreción ${ }^{(1)}$. El daño producido en el hígado es una complicación de casi todos los medicamentos prescritos. Los resultados de la hepatotoxicidad resultan de la toxicidad directa de los compuestos primarios, sus metabolitos reactivos o de la respuesta inmune que afecta a los hepatocitos, células epiteliales biliares, y/o vasculatura hepática ${ }^{(2)}$. De acuerdo a la Agencia Europea de Medicamentos (EMA), la toxicidad hepática ha sido una de las razones más frecuentes de reportes de seguridad en farmacovigilancia y causas de retiro de medicamentos aprobados en el mercado ${ }^{(3)}$. Más de 900 drogas han sido implicadas como una causa de daño hepático. Los fármacos representan el 5\% de hospitalizaciones, $10 \%$ casos de por 
hepatitis aguda, 50\% por falla hepática aguda y la enfermedad hepática crónica representa aproximadamente el $2 \%$ en 17 países, con 40000 muertes por año ${ }^{(4)}$.

El maíz morado es rico en antocianinas, sustancia que le da su coloración característica y que ha sido relacionada con efectos antioxidantes, hipolipemiantes e hipotensores ${ }^{(5)}$. Investigaciones farmacognósicas y fitoquímicas reportan compuestos químicos como flavonoides, terpenos, taninos, aceites esenciales, resinas, saponinas, glicósidos, azufre y fósforo, acido salicílico, sales de potasio y sodio, siendo las que destacan las antocianinas, que es un tipo de flavonoide ${ }^{(6)}$. Las antocianinas son los principales componentes del maíz morado, en especial el cianidin-3-glucósido. Las antocianinas han demostrado efecto modulador de radicales libres de oxígeno y diversas citoquinas ${ }^{(7)}$.

El objetivo principal del presente trabajo fue determinar el efecto hepatoprotector del extracto hidroetanólico atomizado de Zea mays L. (maíz morado) en lesiones hepáticas inducidas en ratas.

\section{MÉTODOS}

Se utilizó ratas albinas machos cepa Holtzman, de 10 semanas de edad, con peso promedio $220 \pm 20 \mathrm{~g}$, procedentes del Instituto Nacional de Salud, del local ubicado en el distrito de Chorrillos de la ciudad de Lima, y fueron acondicionados en el Bioterio de la Facultad de Medicina de la Universidad Nacional Mayor de San Marcos, con condicionamiento previo de 48 horas, con agua y alimento ad líbitum, ciclo luz-día de 12 horas y temperatura de 22 a $26^{\circ} \mathrm{C}$. La coronta de maíz Zea mays L. variedad morada fue procesada de la siguiente manera. Se separó los granos de la coronta, la coronta se llevó a sequedad a temperatura menor de 40 ${ }^{\circ} \mathrm{C}$; se la pulverizó en molino, luego fue sometida a maceración en una solución hidroetanólica y, después de ocho días, se filtró y el producto obtenido se sometió a una operación de atomización, resultando un polvo fino denominado extracto hidroetanólico atomizado de Zea mays L, el que se mezcló con maltodextrina (proporción 10:1) para su mejor estabilidad ${ }^{(6)}$. Posteriormente se determinó los metabolitos secundarios presentes en el extracto con reacciones específicas para cada metabolito, según Lock de Ugaz, $1994{ }^{(7)}$.

La inducción de la lesión hepática se realizó mediante modelo experimental, utilizando fenobarbital más tetracloruro de carbono según Samudram y col., $2008^{(8)}$. Se procedió según el diseño experimental: (G1): Control normal, solvente, $3 \%$ de polisorbato de sodio, 4 $\mathrm{mL} / \mathrm{kg}$; (G2): Control fenobarbital 200 mg en agua de beber, por 15 días; luego, por 7 días tetracloruro de carbono de $0,2 \mathrm{~mL} / \mathrm{kg}$ (diluido al 50\% en aceite de oliva), relación 1:1 (T); (G3): T + silimarina $25 \mathrm{mg} / \mathrm{kg}$; (G4): $\mathrm{T}+$ extracto hidroetanólico 500 mg/kg; (G5): T + extracto hidroetanólico $1000 \mathrm{mg} / \mathrm{kg}$; (G6): $\mathrm{CCl}_{4}+$ extracto hidroetanólico $2000 \mathrm{mg} / \mathrm{kg}$.

Para inducir las lesiones hepáticas crónicas, en un inicio se usó el fenobarbital en agua de beber a $0,5 \mathrm{~g} / \mathrm{L}$ de concentración final, durante 15 días, para luego administrar el tetracloruro de carbono $\left(\mathrm{CCl}_{4}\right)$. Luego, se le administró tetracloruro de carbono $\left(\mathrm{CCl}_{4}\right)$ en dosis de $0,2 \mathrm{~mL} / \mathrm{kg}$ (diluido al $50 \%$ en aceite de oliva), relación 1:1, por vía oral durante siete días.

Para la obtención de la muestra se anestesió a los animales y se obtuvo aproximadamente $5 \mathrm{~mL}$ de sangre mediante punción cardiaca, para después centrifugar y obtener el suero. Las variables de estudio fueron: 1) perfil hepático, 2) hepatomegalia, 3) marcador de estrés oxidativo y, 4) estudio histopatológico.

Para determinar el índice hepático (porcentaje del tejido respecto al peso del animal) y porcentaje de incremento del tejido (hepatomegalia), se empleó la siguiente fórmula:

$$
\begin{aligned}
& \% I H=\frac{W_{T e j}}{W_{P A}} \times 100 \\
& \text { Hepatomegalia }=\frac{W_{T e j}-I H_{C N}}{I H_{C N}} \times 100
\end{aligned}
$$

Donde:

- \%IH: índice hepático.

- $\mathrm{W}_{\mathrm{Tjd}}$ : peso del tejido (hígado).

- $\mathrm{W}_{\mathrm{PA}}$ : peso del animal.

- $\mathrm{IH}_{\mathrm{Tto}}$ : índice hepático del grupo: control negativo, silimarina o los extractos.

- $\mathrm{IH}_{\mathrm{CN}}$ : índice hepático del control negativo.

El perfil hepático se evaluó mediante las enzimas séricas transaminasas (aspartato aminotransferasa, AST, y alanina aminotransferasa, ALT), colesterol total, HDL-C, triglicéridos según el método de Reitman $\mathrm{S}$ y Frankel $\mathrm{F}^{(11)}$; fosfatasa alcalina (FAL) mediante el método propuesto por Bowers GN y McComb RB ${ }^{(12)}$.

La actividad antioxidante se determinó en suero al último día del experimento siguiendo la técnica de Buege y col., 1978, midiendo la producción de especies reactivas al ácido tiobarbitúrico (TBARS) que al reaccionar con el ácido tiobarbitúrico (TBA) forma un complejo coloreado que se lee a 535 nm. Para los cálculos se utilizó el coeficiente de extinción molar $1,56 \times 10^{5}$ $\mathrm{mol}^{-1} \times \mathrm{L} \mathrm{x} \mathrm{cm}^{-1}$ del complejo coloreado formado por el malondialdehído ${ }^{(13)}$.

Una vez sacrificados los animales al administrar pentobarbital $100 \mathrm{mg} / \mathrm{kg}$ por sobredosis, se procedió a realizar cortes de hígado de 1,0 x 0,5 x 1,0 cm de espesor, los que fueron fijados en formol neutro al $10 \%$, siendo seccionados para inclusión en porciones de $2 \mathrm{~mm}$ de espesor. Posteriormente, se efectuó cortes con micrótomo, en un espesor de 3 a 5 micras, para luego ser coloreados con HE (hematoxilina-eosina) y revisados con microscopio óptico ${ }^{(14)}$.

Se realizó la prueba de normalidad mediante la prueba de Shapiro Wilk $(p<0,05)$. Las diferencias entre los 
grupos fueron determinadas mediante análisis de varianza y las comparaciones entre grupos por las pruebas de Anova y post test de Tukey, siendo los resultados significativos con un valor $\mathrm{p}<$ 0,05; se utilizó el programa estadístico SPSS versión 19,0.

\section{RESULTADOS}

Los hallazgos del estudio fitoquímico preliminar mostraron una gran cantidad de compuestos fenólicos, flavonoides y glicósidos caracterizados mediante las pruebas de tricloruro férrico, de Shinoda y de Molish, respectivamente, en el extracto hidroalcohólico atomizado del maíz morado (tabla 1).

El efecto hepatoprotector del extracto hidroalcohólico atomizado del maíz morado fue evaluado al observar los cambios del peso corporal e indicadores bioquímicos: colesterol total, colesterol HDL, triglicéridos, fosfatasa alcalina, ALT y AST. En la tabla 2 se muestra los cambios sobre el perfil hepático, donde se aprecia el incremento de colesterol total, triglicéridos, fosfatasa alcalina y ALT, también disminución de colesterol HDL producidos por el tóxico inductor (fenobarbital más tetracloruro de carbono); y los tratamientos con silimarina y extracto hidroalcohólico atomizado de maíz morado mejoraron estos desniveles. El tetracloruro de carbono aumentó el ALT y redujo la HDL. Se ha evidenciado una reducción de $73 \%$ de TBARS a una dosis de $2000 \mathrm{mg} / \mathrm{kg}, 83 \%$ a dosis de $1000 \mathrm{mg} /$ $\mathrm{kg}$ y $46 \%$ a dosis de $500 \mathrm{mg} / \mathrm{kg}$, como indicador del marcador de estrés oxidativo, como se muestra en la figura 1. El tratamiento con el inductor tóxico en el grupo II indujo al incremento del tejido hepático, tal como los demuestra al compararlo con el grupo I, llegando a ser significativo. El grupo III (silimarina + tetracloruro de carbono) presentó un incremento de tejido hepático de solo $3,1 \%$, respecto al grupo I. Sin embargo, los grupos IV y V mostraron reducción de la masa hepática de 4,8 \% y - $10,1 \%$, respectivamente, tal como se muestra en la tabla 3, siendo esta disminución

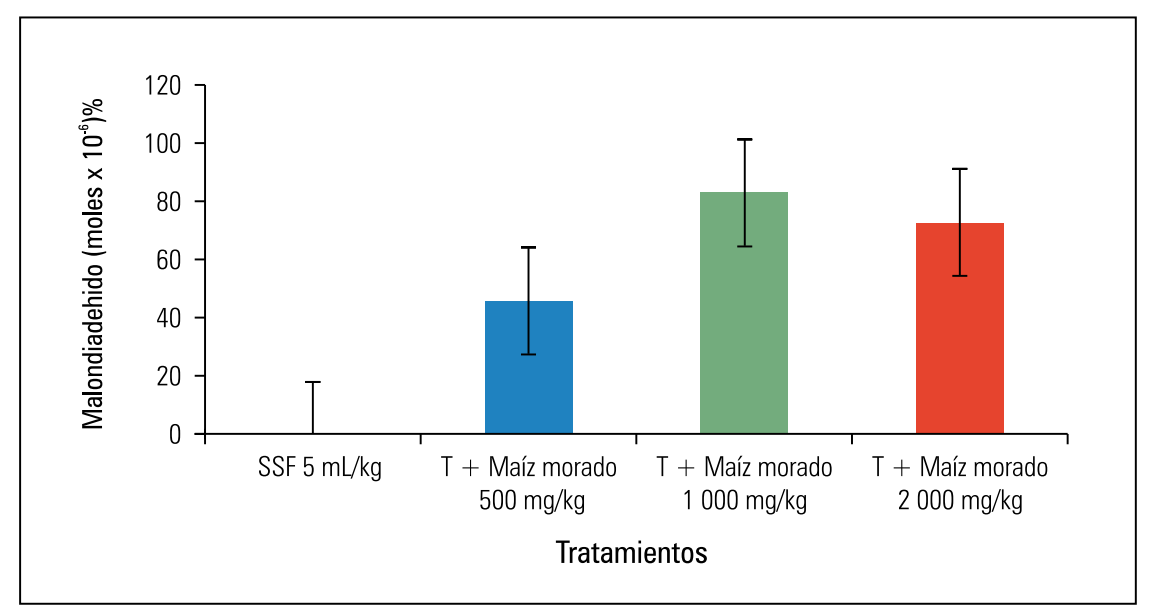

Figura 1. Porcentaje de variación del especies reactivas al ácido tiobarbitúrico (TBARS) en ratas con lesión hepática inducida por tetracloruro de carbono y tratadas con Zea mays L.

significativa $(\mathrm{p}<0,001)$. El colágeno, la fibrosis y el nivel de daño hepático aumentaron con fenobarbital- tetracloruro de carbono; estos indicadores se redujeron con los diferentes tratamientos de maíz morado e incluso silimarina.

\section{DISCUSIÓN}

Las lesiones hepáticas se explicarían porque el fenobarbital es un potente estimulante hepático que in vivo produce hipertrofia e hiperplasia a este nivel; del mismo modo, otros carcinógenos celulares frecuentemente son usados en este tipo de modelos experimentales. Asimismo, se ha comunicado que el tratamiento a largo plazo en ratas resulta en atrofia, disminución y pérdida de regeneración hepática ${ }^{(15)}$. En cambio, el tetracloruro de carbono $\left(\mathrm{CCl}_{4}\right)$

se metaboliza activamente por el citocromo $\mathrm{P} 450$ al radical triclorometilo, el cual inicia la lipoperoxidación celular, produciendo daño hepático al comprometer la integridad de las membranas y por la unión covalente de intermedios reactivos a moléculas biológicamente importantes, como el glutatión, induciendo necrosis y daño hepático en general ${ }^{(16)}$. Por ello, se considera al estrés oxidativo como el principal mecanismo molecular involucrado en la toxicidad por tetracloruro de carbono, el cual tiene rol importante para la inactivación de células Kupffer en la fibrosis hepática inicial inducida por este agente ${ }^{(17,18)}$. La producción de radicales libres durante el desarrollo del daño hepático conduce a la disminución de la actividad de la superóxido dismutasa. En ratas con inducción de cirrosis por $\mathrm{CCl}_{4}$, se ha

Tabla 1. Prueba preliminar cualitativa de los metabolitos secundarios del extracto hidroalcohólico atomizado de maíz morado (Zea mays L.).

\begin{tabular}{ccc} 
Reactivo & Resultado & Metabolito \\
Ninhidrina & $(-)$ & Aminoácidos libres \\
Gelatina & $(-)$ & Taninos \\
Cloruro férrico $(\mathrm{FeCl} 3)$ & $(+++)$ & Compuestos fenólicos \\
Dragendörff & $(+)$ & Alcaloides \\
Mayer & $(+)$ & Alcaloides \\
Shinoda & $(+++)$ & Flavonoides \\
\hline
\end{tabular}

$(-)=$ Ausencia; $(+)=$ Poca cantidad; $(++)=$ Regular cantidad; $(+++)=$ Bastante cantidad . 
Tabla 2. Valores medios y porcentajes de variación del perfil hepático en ratas.

\begin{tabular}{|c|c|c|c|c|}
\hline Variable & Tratamiento & Media & $\mathrm{EE}$ & $\% \operatorname{Var}$ \\
\hline \multirow{6}{*}{$\begin{array}{l}\text { Colesterol total } \mathrm{mg} / \mathrm{dL} \\
\qquad p=0,218\end{array}$} & Normal & 163,2 & 25,3 & 0,0 \\
\hline & Tetracloruro (T) & 181,6 & 45,8 & 10,1 \\
\hline & $\mathrm{T}+$ Silimarina $25 \mathrm{mg} / \mathrm{kg}$ & 154,8 & 35,3 & $-5,4$ \\
\hline & $\mathrm{T}+$ Extracto $500 \mathrm{mg} / \mathrm{kg}$ & 174,6 & 29,4 & 6,5 \\
\hline & $\mathrm{T}+$ Extracto $1000 \mathrm{mg} / \mathrm{kg}$ & 160,2 & 45,3 & $-1,9$ \\
\hline & $\mathrm{T}+$ Extracto $2000 \mathrm{mg} / \mathrm{kg}$ & 151,5 & 25,9 & $-7,7$ \\
\hline \multirow{6}{*}{$\begin{array}{l}\mathrm{HDL} \mathrm{mg} / \mathrm{dL} \\
p=0,385\end{array}$} & Normal & 52,9 & 12,1 & 0,0 \\
\hline & Tetracloruro (T) & 50,1 & 20,1 & $-5,6$ \\
\hline & $\mathrm{T}+$ Silimarina $25 \mathrm{mg} / \mathrm{kg}$ & 54,8 & 14,8 & 3,4 \\
\hline & $\mathrm{T}+$ Extracto $500 \mathrm{mg} / \mathrm{kg}$ & 49,5 & 16,8 & $-6,9$ \\
\hline & $\mathrm{T}+$ Extracto $1000 \mathrm{mg} / \mathrm{kg}$ & 48,3 & 21,8 & $-3,7$ \\
\hline & $\mathrm{T}+$ Extracto $2000 \mathrm{mg} / \mathrm{kg}$ & 45,6 & 20,3 & $-16,0$ \\
\hline \multirow{6}{*}{$\begin{array}{l}\text { Triglicéridos mg/dL } \\
\qquad p=0,485\end{array}$} & Normal & 98,5 & 15,6 & 0,0 \\
\hline & Tetracloruro (T) & 134,1 & 21,3 & 26,5 \\
\hline & $\mathrm{T}+$ Silimarina $25 \mathrm{mg} / \mathrm{kg}$ & 135,4 & 19,5 & 27,2 \\
\hline & $\mathrm{T}+$ Extracto $500 \mathrm{mg} / \mathrm{kg}$ & 140,6 & 25,3 & 30,0 \\
\hline & $\mathrm{T}+$ Extracto $1000 \mathrm{mg} / \mathrm{kg}$ & 130,4 & 26,1 & 24,4 \\
\hline & $\mathrm{T}+$ Extracto $2000 \mathrm{mg} / \mathrm{kg}$ & 143,6 & 24,6 & 31,4 \\
\hline \multirow{6}{*}{$\begin{array}{c}\text { Aspartato amino } \\
\text { transferasa (AST) U/L } \\
\quad p=0,054\end{array}$} & Normal & 13,5 & 5,3 & 0,0 \\
\hline & Tetracloruro (T) & 25,1 & 6,1 & 46,2 \\
\hline & $\mathrm{T}+$ Silimarina $25 \mathrm{mg} / \mathrm{kg}$ & 11,9 & 2,3 & $-13,4$ \\
\hline & $\mathrm{T}+$ Extracto $500 \mathrm{mg} / \mathrm{kg}$ & 13,5 & 4,4 & 0,0 \\
\hline & $\mathrm{T}+$ Extracto $1000 \mathrm{mg} / \mathrm{kg}$ & 10,6 & 3,8 & $-27,4$ \\
\hline & $\mathrm{T}+$ Extracto $2000 \mathrm{mg} / \mathrm{kg}$ & 17,8 & 4,6 & 24,1 \\
\hline \multirow{6}{*}{$\begin{array}{c}\text { Alanina amino } \\
\text { transferasa (ALT) U/L } \\
p=0,084\end{array}$} & Normal & 35,5 & 10,3 & 0,0 \\
\hline & Tetracloruro (T) & 39,8 & 16,5 & 10,8 \\
\hline & $\mathrm{T}+$ Silimarina $25 \mathrm{mg} / \mathrm{kg}$ & 42,9 & 14,7 & 17,2 \\
\hline & $\mathrm{T}+$ Extracto $500 \mathrm{mg} / \mathrm{kg}$ & 13,5 & 4,4 & 0,0 \\
\hline & $\mathrm{T}+$ Extracto $1000 \mathrm{mg} / \mathrm{kg}$ & 34,3 & 9,8 & $-3,4$ \\
\hline & $\mathrm{T}+$ Extracto $2000 \mathrm{mg} / \mathrm{kg}$ & 38,9 & 16,7 & 8,7 \\
\hline \multirow{6}{*}{$\begin{array}{l}\text { Fosfatasa alcalina } \\
\text { (FAL) U/L } \\
p=0,064\end{array}$} & Normal & 115,3 & 35,8 & 0,0 \\
\hline & Tetracloruro (T) & 125,1 & 41,6 & 7,8 \\
\hline & $\mathrm{T}+$ Silimarina $25 \mathrm{mg} / \mathrm{kg}$ & 93,5 & 35,3 & $-23,3$ \\
\hline & $\mathrm{T}+$ Extracto $500 \mathrm{mg} / \mathrm{kg}$ & 119,6 & 25,5 & 3,6 \\
\hline & $\mathrm{T}+$ Extracto $1000 \mathrm{mg} / \mathrm{kg}$ & 117,5 & 35,4 & 1,9 \\
\hline & $\mathrm{T}+$ Extracto $2000 \mathrm{mg} / \mathrm{kg}$ & 114,2 & 34,6 & $-0,9$ \\
\hline
\end{tabular}

$\mathrm{EE}=$ error estándar

$\% \operatorname{Var}=$ Porcentaje de variación $=[($ Valor medio del tratamiento X 100) $/$ Valor medio del normal $)-100)]$.

encontrado correlación inversa entre las enzimas antioxidantes y las puntuaciones patológicas y/o de peroxidación lipídica ${ }^{(19)}$.

Se identificó la presencia de metabolitos secundarios, tales como aminoácidos libres, taninos, alcaloides, compuestos fenólicos y flavonoides, siendo estos últimos los de mayor cantidad (tabla 1). Es conocido que la mayoría de los flavonoides son poderosos antioxidantes y poseen diversas activi- dades antiinflamatorias; los flavonoides son inhibidores de la formación de leucotrieno B4, potenciadores de la formación de prostaglandina E2 e inhibidores de la liberación de óxido nítrico ${ }^{(20)}$. Así como efecto hepatoprotector relacionado con la capacidad de estos de disminuir el estrés oxidativo y atrapar radicales libres, tanto in vivo como in vitro; un ejemplo es el caso de la quercetina, la cual ha demostrado ser efectiva contra el daño hepático en ratas con inducción de cirrosis con tetracloruro de carbono, asociado con un incremento de la capacidad antioxidante del hígado para atrapar radicales peroxilo; y en ratas con obstrucción biliar crónica, el tratamiento con quercetina resultó en una preservación significativa de la actividad de las enzimas antioxidantes, fibrosis menos pronunciada y marcada inhibición de la proliferación ductular biliar ${ }^{(21)}$.

En el presente trabajo se ha demostrado la reducción del marcador del estrés oxidativo (malondialhedido) como indicador del efecto antioxidante encontrado. Se justifica posiblemente por la presencia de compuestos fenólicos, como lo expresan quienes encontraron un incremento de la actividad antioxidante del plasma en ratas que reciben compuestos fenólicos, resultados muy semejantes a los que se obtuvo con el extracto hidroalcohólico atomizado de Zea mays L. Al evaluar diferentes marcadores bioquímicos, no hubo diferencia significativa entre los grupos de tratamiento que recibieron el tóxico como la silimarina y las diferentes dosis del extracto hidroetanólico atomizado de maiz morado. Sin embargo, sí hubo diferencia en el peso del hígado (tabla 3); en los animales con lesiones hepáticas fue significativamente menor comparado con los animales del grupo control y los otros grupos de tratamiento que recibieron el extracto de maíz morado, mostrando una disminución del índice de hepatomegalia $(p<0,001)$ causado por la administración del tóxico; este efecto podría estar asociado a alteraciones en la absorción de nutrientes y la utilización metabólica ${ }^{(22)}$.

Las antocianinas son los elementos más reconocido del grupo de los flavonoides. Bell y col. ${ }^{(23)}$ han demostrado que son potentes antioxidantes y capturadores excepcionalmente potentes de radicales libres de oxígeno, los cuales tienen un rol crítico en la patogénesis de la enfermedad hepáticas. Estos hallazgos se complementan bien con la buena tasa de absorción y biodisponibilidad que tienen las antocianinas presentes en el Zea mays L., tanto en ratas como en humanos; en especial, 
Tabla 3. Índice hepático e incremento de tejido (hepatomegalia).

\begin{tabular}{ccc} 
& Índice hepático & Hepatomegalia \\
Grupo I & $4,50 \pm 0,11$ & - \\
Grupo II & $4,95 \pm 0,25$ & 6,2 \\
Grupo III & $4,40 \pm 0,25$ & 4,5 \\
Grupo IV & $4,57 \pm 0,35$ & 4,8 \\
Grupo V & $4,25 \pm 0,38^{a}$ & $-10,1$ \\
Grupo VI & $4,63 \pm 0,85$ & 5,6 \\
\hline
\end{tabular}

(a) Comparado con el grupo control positivo $p<0,001$.

la cianidina 3 glicósido, la cual, tras ser administrada por vía oral, es incorporada a la circulación, manteniendo su estructura química intacta. Aún falta determinar la farmacocinética de estos compuestos, su toxicidad, relevancia clínica y limitaciones; así como determinar si el consumo tradicional de la 'chicha morada' en nuestro medio llega a ser un factor de protección en la salud pública ${ }^{(24)}$.
Con respecto al dosaje de enzimas hepáticas y proteínas totales, los niveles séricos de alanina amino transferasa (ALT) en las ratas sin insuficiencia hepática se encuentran en el límite superior de los valores normales (tabla 2), y en las ratas con insuficiencia hepática estuvieron incrementados; esto ocurre con sustancias como la D. galactosamina que induce insuficiencia hepática ${ }^{(25)}$. En la figura 2, en el grupo tratado con tetracloruro de carbono se

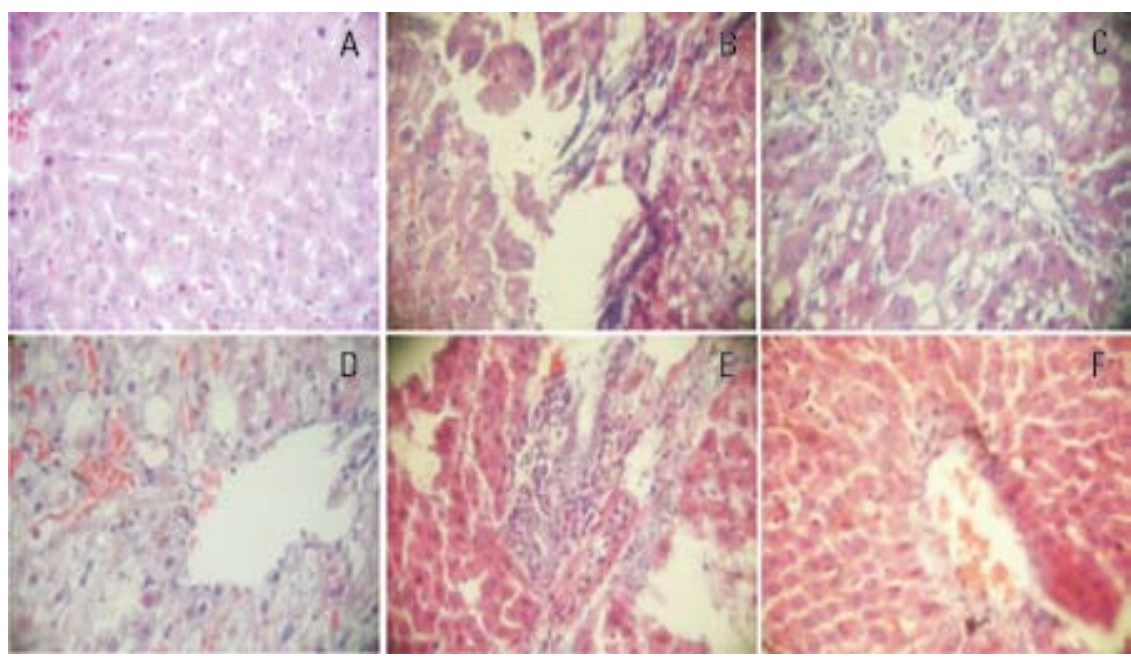

Figura 2. Microfotografías de hígado de ratas tratadas con el extracto atomizado de Z. mays en un modelo de lesión hepática inducida por tetracloruro de carbono (CCl4). 400 X. A: Grupo control negativo; tejido hepático normal. B: Grupo tratado con $\mathrm{CCl} 4$; daño hepático con alteraciones en formas de fibrosis, habiendo variaciones de microvacuolas hasta adipocitos en el espacio de Kier. C: Grupo tratado con silimarina; mejora en las estructuras de los hepatocitos, con núcleo en cariolisis y cariorrexis, e infiltraciones. D: Grupo tratado con Z. mays $500 \mathrm{mg} / \mathrm{kg}$, esteatosis, reemplazo de células hepáticas por adipocitos, espacio de Kier con discreta alteración. E: Grupo tratado con Z. mays $1000 \mathrm{mg} / \mathrm{kg}$; hepatocitos conservados, con estructuras hepáticas conservadas. Hepatocitos con discreta pigmentación citoplasmáticas glandulares; $50 \%$ de núcleos conservados; espacio de Kier con hipertrofia en la base de las células de Kupffer e histiocitos. F: Grupo tratado con Z. mays 2000 mg/kg; se observa congestión en venas centrolubulillares, espacio de Kier con presencia de histiocitos y fibrocitos; células de Kupffer y macrófagos han fagocitado pigmentos oscuros; mayor de $50 \%$ de núcleos conservados. evidenció incremento de la fibrosis en el colágeno, y por tanto el dañó hepático; la fibrosis hepática es la consecuencia común a lesiones hepáticas crónicas de muchas etiologías. El abuso crónico de alcohol es el principal motivo de que aparezca fibrosis hepática, lo que conduce a la cirrosis, una de las principales causas de muerte en todo el mundo. La fibrosis se caracteriza por una acumulación excesiva de proteínas de matriz extracelular (MEC), tal como el colágeno de tipo I (encontrado en humanos), que se encuentran normalmente en la zona pericentral y perisinusoidal del hígado ${ }^{(26)}$.

Se concluye que el extracto hidroetanólico atomizado de maiz morado (Zea mays L.) tuvo efecto hepatoprotector sobre las lesiones hepáticas inducidas en ratas, a la dosis de $1000 \mathrm{mg} / \mathrm{kg}$.

\section{AGRADECIMIENTOS}

Nuestro agradecimiento al laboratorio FITOFARMA EIRL. por la elaboración del extracto hidroetanólico atomizado de maíz morado (Zea mays L.) en sus instalaciones.

\section{REFERENCIAS BIBLIOGRÁFICAS}

1. Singh A, Bhat TK, Sharma OP. Clinical biochemistry of hepatotoxicity. J Clinic Toxicol. 2011;S4:001.

2. Pandit A, Sachdeva T, Bafna P. Drug-induced hepatotoxicity: a review. J Appl Pharm Sci. 2012;2(5):233-43.

3. European Medicines Agency (EMEA). Non-clinical guideline on drug-induced hepatotoxicity. London: EMEA (Committee for Medicinal Products for Human Use [CHMP]), Doc. Ref. EMEA/CHMP/ SWP/150115/2006, 2008: 1-16.

4. Navarro VJ, Senior JR. Drug-related hepatotoxicity. N Engl J Med. 2006;354:731-9.

5. Andlauer W, Stumpf C, Frank K, Fürst P. Absorption and metabolism of anthocyanin cyanidin-3-glucoside in the isolated rat small intestine is not influenced by ethanol. Eur J Nutr. 2005;42(4):217-33.

6. Arroyo J, Ráez E, Rodríguez M, Chumpitaz V, Burga J, De la Cruz W y col. Reducción del colesterol y aumento de la capacidad antioxidante por el consumo crónico de maiz morado (Zea mays L) en ratas hipercolesterolémicas. Rev Peru Med Exp Salud Publica. 2007;24(2):157-62.

7. Arroyo J, Ráez E, Rodriguez M, Chumpitaz V, Burga J, De la Cruz Wy col. Actividad antihipertensiva y antioxidante del extracto hidroalcohólico atomizado de maiz morado (Zea mays $L$ ) en ratas. Rev Peru Med Exp Salud Publica. 2007;24(2):157-82. 
8. Lock O. Investigación fitoquímica. Métodos en el estudio de productos naturales. 2da ed. Lima: Fondo Editorial Pontificia Universidad Católica del Perú. 1994;1-7.

9. Samudram P, Hari R, Vasuki R, Geetha A, Moorthi SP. Hepatoprotective activity of bi-herbal ethanolic extract on $\mathrm{CCl} 4$ induced hepatic damage in rats. Afr J Biochem Res. 2008;2(2):61-5.

10. Frankel S, Reitman S, Sonnenwirth AC, editors. Gradwohl's Clinical Laboratory Methods and Diagnosis. $7^{\text {a }}$ Ed. Vol. 1. Saint Louis: The C. V. Mosby Company; 1970.

11. Bowers GN, McComb RB. Measurement of tota alkaline phosphatase activity in human serum. Clin Chem. 1975;21(13):1988-95.

12. Gabor S, Bergmeyer HU. Methods of Enzymatic

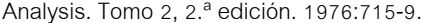

13. Buege JA, Aust SD. Microsomal lipid peroxidation. Methods Enzymol. 1978;52:302-10

14. Devi RS, Narayan S, Vani G, Shyamala Devi CS. Gastroprotective effect of Terminalia arjuna bark on diclofenac sodium induced gastric ulcer. Chem Biol Interact. 2007;167(1):71-83

15. Cho YK, Yun JW, Park JH, Kim HJ, Park DI, Sohn Cl, et al. Deleterious effects of silymarin on the expression of genes controlling endothelial nitric oxide synthase activity in carbon tetrachloride treated rat livers. Life Sci. 2009;85(7-8):281-90.

16. Wang L, Cheng D, Wang H, Di L, Zhou X, Xu T, Yang X, Liu Y. The hepatoprotective and antifibrotic effects of Saururus chinensis against carbon tetrachloride induced hepatic fibrosis in rats. Ethnopharmacol. 2009;126(3):487-91.

17. Perez Gutierrez RM, Anaya Sosa I, Hoyo Vadillo C, Victoria TC. Effect of flavonoids from Pros thechea michuacana on carbon tetrachloride induced acute hepatotoxicity in mice. Pharm Biol. 2011:49(11):1121-7.

18. Polyak SJ, Morishima C, Lohmann V, Pal S, Lee DY, Liu Y, Graf TN, Oberlies NH. Identification of hepatoprotective flavonolignans from silymarin. Proc Natl Acad Sci U S A. 2010;107(13):5995-9.

19. Kviecinski MR, Felipe KB, Correia JF, Ferreira EA, Rossi MH, de Moura Gatti F, Filho DW, Pedrosa RC. Brazilian Bidens pilosa Linné yields fraction containing quercetin-derived flavonoid with free radical scavenger activity and hepatoprotective effects. Libyan J Med. 2011;6. doi: 10.3402/ljm. v6i0.5651.

20. Moreno O, Paz-Aliaga A. Efecto vasodilatador mediado por óxido nítrico del extracto hidroalcohólico de Zea mays I. (maíz morado) en anillos aórticos de rata. Rev Peru Med Exp Salud Publica. 2010;27(4):527-31.

21. Rocha C, Bolognesi V, Gaspari J, Gomes O, Klocker O. Carotenoides bixina e norbixina extraídos do urucum (Bixa orellana L.) como antioxidantes em produtos cárneos. Ciência Rural, Santa Maria. 2012;42(8):1510-7.

22. Sakuragui M. Indução do sistema citocromo P450 em linhagens de hepatoma humano para utilização como modelo in vitro no desenvolvimento de fármacos. [Tese para obtençãu do grau de Doctor] São Paulo 2011.

23. Bell DR, Gochenaur K. Direct vasoactive and vasoprotective properties of anthocyanin-rich extracts. J Appl Physiol. 2006;100(4):1164-70.

24. Favari-Perozzi L, Nava-Álvarez R, MeléndezCamargo ME. Probable efecto hepatoprotector de la verbena en la hepatitis inducida con tetra- cloruro de carbono en la rata. Rev Mex cienc farm. 2007;38(3):19-25.

25. Adewusi E, Afolayan A. A review of natural products with hepatoprotective activity. J Medicinal Plants Res. 2010;4(13):1318-34.

26. Sabir SM, Rocha BTJ. Water-extractable phytochemicals from Phyllanthus niruri exhibit distinct in vitro antioxidant and in vivo hepatoprotective activity against paracetamol-induced liver damage in mice. Food Chemistry. 2008;111(4):845-51.

Artículo recibido el 15 de setiembre de 2014 y aceptado para publicación el 22 de octubre de 2014.

Conflictos de intereses.

Los autores expresan no tener algún conflicto de interés

Correspondencia:

Mg. Oscar Herrera Calderon

Dirección: Ciudad Universitaria (Ica, Panamericana Sur Km 305 s/n) Universidad Nacional San Luis Gonzaga de Ica, Departamento Académico de Ciencias Farmacéuticas.

Teléfono: $956550510-+51+56+213050$

Correo electrónico: oh2000_4@hotmail.com 